\title{
New Cavity Backed Capacitively Coupled Stacked Patch Element for P-Band SAR Application
}

\author{
Markus Limbach, B. Gabler, R. Horn, A. Kosc, A. Di Maria \\ ${ }^{1}$ SAR-Technology, Microwaves and Radar Institute, DLR, Oberpfaffenhofen, Germany, markus.limbach@dlr.de
}

\begin{abstract}
The Microwaves and Radar Institute at the German Aerospace Center operate an airborne multi-frequency, polarimetric, imaging SAR system. The F-SAR sensor is equipped with a variety of different antennas, based on patch technology. A new antenna element is developed for the P-Band frequency range from 400 to $470 \mathrm{MHz}$. Due to the requirements of the aircraft the size of the antenna array and therefore of each single element, is reduced to a minimum. A cavity backed, capacitively coupled stacked patch element is designed and presented in this paper.
\end{abstract}

Index Terms - patch antenna, SAR sensor, cavity backed, pband.

\section{INTRODUCTION}

Imaging radar systems are widely used in earth observation purposes, either operated on spaceborne or airborne platforms. For high resolution products synthetic aperture radar (SAR) sensors are most suitable. The resolution in direction of motion is limited by the physical length of the antenna, thus small antennas are preferred.

The response of a radar pulse depends strongly on the frequency range. Additional information can be gained by the usage of different wavelengths in one observation area. The FSAR system, operated by the Microwaves and Radar Institute at German Aerospace Center in Oberpfaffenhofen, is equipped with five frequency ranges. Starting at X-band frequencies the system carries also C, S, L and P-band subsystems. For the Pband a new antenna is developed, covering the range from 400 to $470 \mathrm{MHz}$. The airborne platform, a Dornier Do 228 and the mounting area underneath the fuselage, gives the maximum physical dimensions for the P-band antenna array. The envelope is about $1.4 \mathrm{~m} \times 1.4 \mathrm{~m} \times 0.4 \mathrm{~m}$ with a maximum weight of $100 \mathrm{~kg}$.

The mounting area underneath the aircrafts fuselage requires an electrical beam pointing at 40 degree to the righthand side. For proper opposite swath and nadir signal suppression a minimum number of antenna elements in lateral direction is essential. At least five elements are needed. At the given antenna size an electrically small array results.

A single, slot coupled, patch element design in this frequency range was already presented, together with the network power dividers [1]. Setting up the antenna array, strong coupling effects in-between this type of radiating elements makes their usage in the electrically small array unfeasible. With a coupling of about $-6 \mathrm{~dB}$, see Fig.1, the influence of adjacent elements is extremely high. The side looking configuration is distorted by the overlay of amplitude and phase taper settings with the coupling vectors. Strong variations over frequency in beam shape and pointing of the main beam direction occurs, beneath low radiation efficiency. To overcome the coupling effects an antenna element is designed with metallic walls to the neighboring element. Consequently a resonator is generated with metallic walls and ground plane. Inserting patches and a feeding structure a wideband antenna can be formed [2], [3]. Various layouts were tested to meet the requirements of the SAR system with dual polarizations and wideband operation. The dimensions of the resonator are the most critical component in the design.

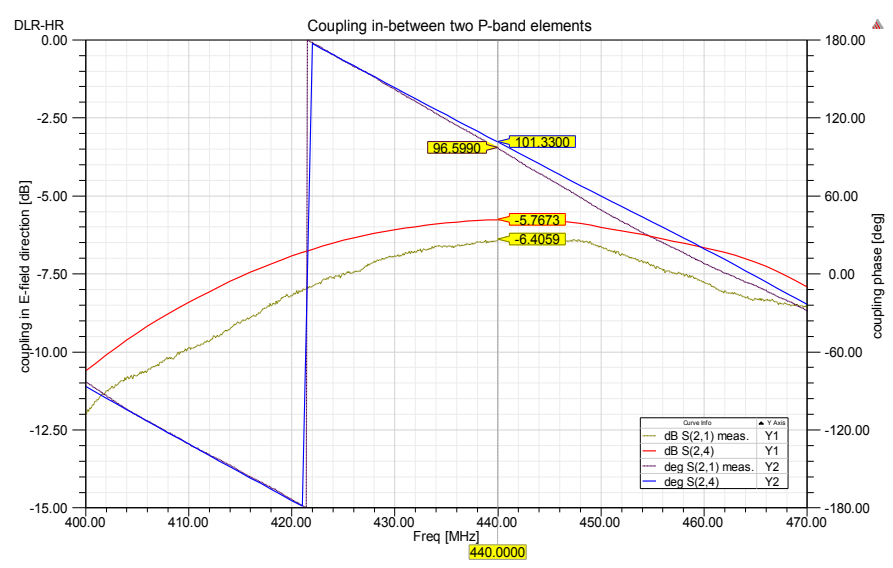

Fig. 1. Coupling effect for two adjacent patch elements in E-plane direction. Simulation and measured data in amplitude and phase.

\section{SPECIFICATION}

The F-SAR system is a modular, experimental airborne SAR sensor, designed for flexible operations. It can be adjusted to new radar modes and frequency ranges, acting like a test bed for new algorithms and future SAR systems in orbit.

The new frequency range for spaceborne earth observation at $435 \mathrm{MHz}$ is not yet covered by the F-SAR system. The current P-band design starts at $300 \mathrm{MHz}$ and ends at $400 \mathrm{MHz}$, where the recent range will initiate. A new antenna in conjunction with additional radar front-end shall enhance the F-SAR capabilities to the higher frequencies. The new P-band specification covers the range from 400 to $470 \mathrm{MHz}$.

The antenna operates in two modes, a nadir looking sounder configuration and the standard side looking SAR configuration with an electrical shift of the main beam at 40 degree off nadir direction. In side looking mode the antenna 
main beam covers the area from 25 degree off nadir up to 60 degree. With the antenna mounted underneath the aircraft's fuselage it is necessary to suppress any radiation other than the desired angular range, especially in nadir and opposite swath direction. A sufficient number of elements in lateral direction is necessary. With an element spacing of $1 / 2$ of a wavelength at center frequency $(350 \mathrm{~mm})$ in a $5 \times 5$ element array the available space underneath the airplanes fuselage would be more than totally consumed. With only $1.4 \mathrm{~m}$ available space the element size has to be reduces by $20 \%$, to $280 \mathrm{~mm}$ cell size.

The maximum pulse power for the antenna array is $1 \mathrm{~kW}$ with $10 \%$ duty cycle. The first stage of the power dividing network is most affected by the pulse power. At a flight altitude of $3000 \mathrm{~m}$ up to $6000 \mathrm{~m}$ the components must be designed to withstand the thermal dissipation of the electrical losses as well as the high voltage of the peak power and voltage standing wave ratio.

The mechanical structure of the antenna array has to meet all airworthiness aspects like lightning protection, static weight, dynamic weight (50 feet gust) and forces due to the airflow. A wind shield, to prevent the structure from direct wind impact, exists already and one of its mechanical interfaces is located on the aperture of the new antenna array.

\section{ANTENNA ELEMENT DESIGN}

The standard patch element design, stacked and or slot coupled, is used for most of the F-SAR antennas. Due to the small array size at P-band and the beam pointing over more than $16 \%$ band width, this type of radiating elements is unfeasible. The radiating areas of the patch elements, the magnetic walls, are very close to each other, thus the electromagnetic coupling is strong, in the range of only $-6 \mathrm{~dB}$. It is not possible, pointing the main beam to the dedicated position and shape the diagram proper for all frequencies. In addition the efficiency of the antenna array would be far below $60 \%$.

To overcome the strong coupling effects the radiating elements can be moved away from each other. In consequence the array will grow in size or elements have to be neglected. Both possibilities are out of the specification.

To weaken the electromagnetic coupling the direct path between adjacent elements has to be suppressed. Based on the cavity model of the patch element the radiating area is at the edge of the patch among the patch and the ground plane. With a conducting structure in that area the electrical field vector can be controlled. A metal wall is than inserted in-between the radiating areas of the patch elements; the conducting structure forces the tangent component of the electric field vector to null and minimizes the coupling effects. The result is a resonator backed patch element [2].

A number of band width enhancement methods are published for this type of antenna structure. They bases on regular resonator dimensions and show more than $25 \%$ feasible bandwidth for dual polarized elements. The design goal here is to reduce the resonator size and simultaneously enhance the bandwidth performance. These requirements are antipodal and additional components have to be inserted in the common design.

\section{OPTIMIZATION AND SIMULATION RESULTS}

The specified frequency range of the P-band subsystem is $70 \mathrm{MHz}$ of bandwidth at a center frequency of $435 \mathrm{MHz}$, the wavelength in air is $690 \mathrm{~mm}$. A resonator at $\mathrm{TEM}_{10}$ mode has an optimum length of $\lambda / 2$. According to the available antenna array size and number of elements the maximum available length is $280 \mathrm{~mm}$, already including the necessary structural elements for airworthiness. The clear inside dimensions for the resonator are somewhat smaller.

To cover the required $16 \%$ of bandwidth the design is enhanced with two patch layers. The lower patch is excited by two capacitive feed probes, one for each polarization.

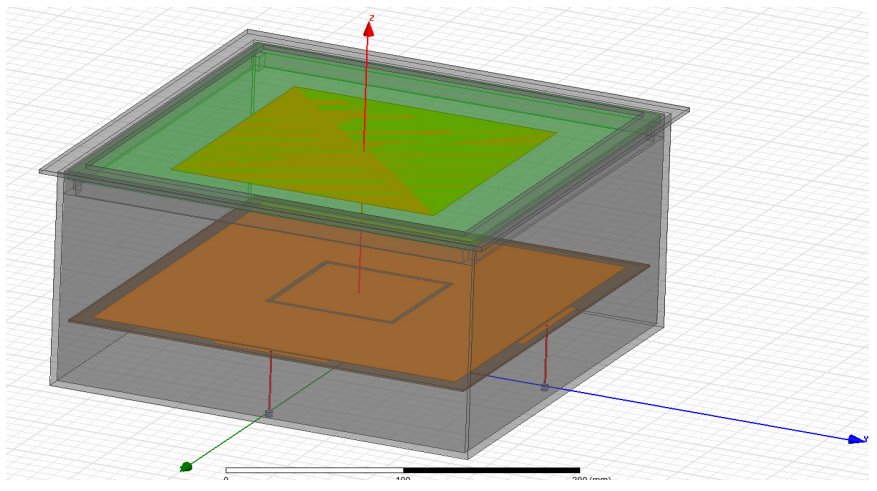

Fig. 2. View of 3-D simulation model from Ansys HFSS V.15

The lower patch is designed on top of a $1.28 \mathrm{~mm}$ thick Rogers RO $3006^{\mathrm{TM}}$ layer. With only about $1 \mathrm{~mm}$ thickness the effect of minimizing the physical length of the patch resonator is negligible. The free resonator height underneath the Rogers layer is $41 \mathrm{~mm}$ to the bottom of the resonator. To feed the patch a rectangular pad is designed; soldered to the center pin of the coaxial line in the bottom plate. The size of the pad and distance to the edge of the patch in conjunction with the diameter of the coaxial center line is used to match the antenna element. The capacitive coupling between patch and pad cancels the inductive part of the $42.28 \mathrm{~mm}$ long center pin.

To control the real part of the input impedance the patch includes a quadratic slot around its center. The real part of the input impedance can be adjusted easily varying the slots dimensions. To hold the lower substrate layer in place it is supported by a block made of Rohacell ${ }^{\odot}$.

A second patch is located underneath a radome layer of $6 \mathrm{~mm}$ FR4 material. This material is mainly used by its mechanical and chemical strength again environmental conditions during operations of the aircraft. The antennas mounting position, close behind the front gear, is not free of gravel impacts. The radome material can easily withstand most of the impacts, but has to have a minimum thickness of $6 \mathrm{~mm}$. It is an important contribution to the antennas weight. The FR4 material is fixed with an aluminium frame. The metal frame on top of each element is simultaneously used for sufficient lightning protection at the antenna aperture and will house the mechanical interface to mount the wind shield. 
The free length inside of the resonator is $258.2 \mathrm{~mm}$ and thus significantly smaller than $\lambda / 2$. For the reasons of bandwidth and mechanical weight the resonator cannot be filled with dielectric material, capable to compensate the physical length due to its dielectric behavior. The single element design has reached its absolute minimum dimensions in $\mathrm{x}-$, $\mathrm{y}$ - and $\mathrm{z}$ - directions.

The Fig. 2 shows the simulation model of the cavity backed, capacitive coupled, stacked patch element. The single antenna element is optimized with the help of Ansys HFSS. The $70 \mathrm{MHz}$ bandwidth requirement, according to $16 \%$ relative bandwidth, is defined at $-10 \mathrm{~dB}$ return loss at the antenna feeding connector. With additional metallic components inside the resonator, used as structural parts in the antenna design, the input impedance changes a little bit to higher frequencies. Fig. 3 shows the simulation results of the single element with and without the structural elements.

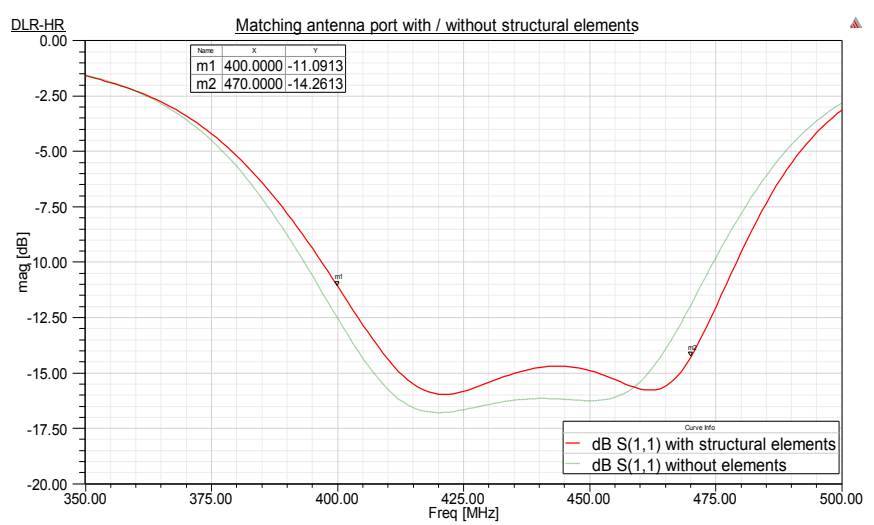

Fig. 3. Input impedance of a single element, with (red) and without (green dotted) structural elements.

The requirements are well achieved by both models, a wider and deeper resonator would give better performance, but size is clipped by the location of the.

\section{ARRAY DESIGN}

The simulation of two antenna elements in the desired array configuration demonstrates the effect of shutting down the direct path. The coupling is reduced by more than $-15 \mathrm{~dB}$ over the frequency range.

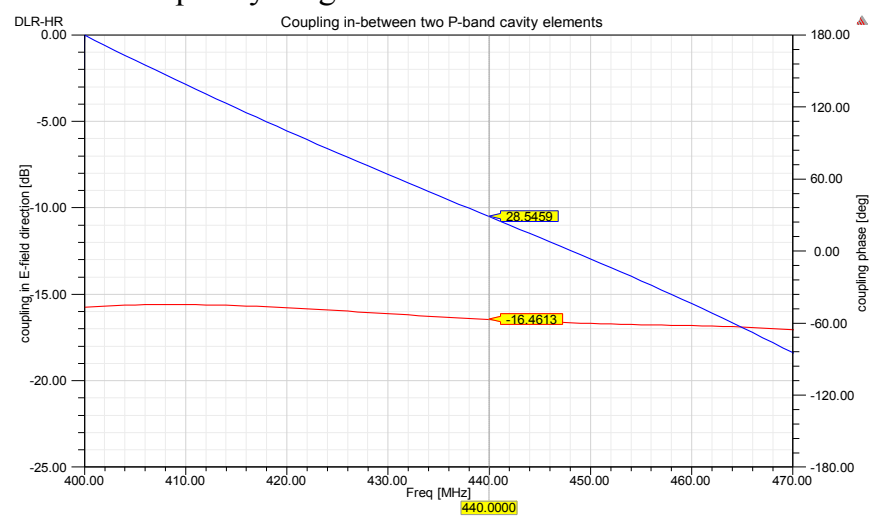

Fig. 4. Coupling effect for two adjacent resonator elements in E-plane direction.
With the new element design we could gain $10 \mathrm{~dB}$ in decoupling behavior, see Fig. 4.

The distribution of the E-field showed for two mirrored elements in Fig. 5, is dominated by the quasi-field-free area inbetween the two elements. In the former patch design this area was the major coupling path in the array. Only fringing fields are feeding the element on the right hand side.

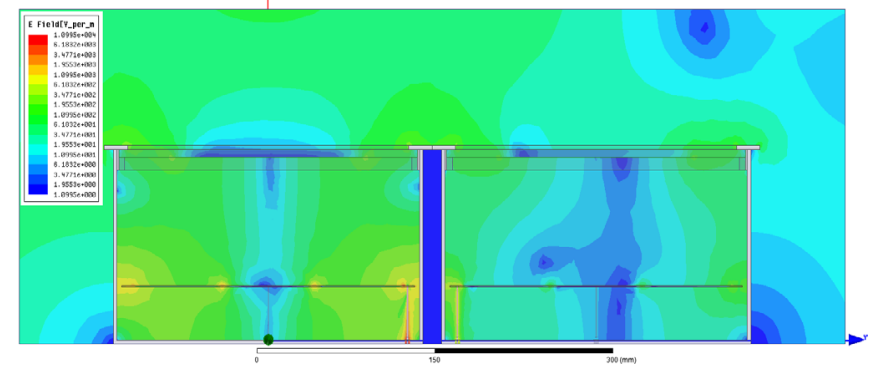

Fig. 5. Illustration of the E-field for two resonator elements. The left element is driven, whereas the right element is passive in the simulation.

To evaluate the design relating the beam steering requirements a row is modeled and calculated in HFSS as a single design, including all five elements in a unique configuration. In simulation the phase and amplitude tapering for the five element ports is optimal in frequency. There is no phase or amplitude deviation over frequency. Using a real power dividing network filter effects and phase variations will occur and have to be adjusted to the antenna array for optimum pattern. The ports phase tapering is 107 degree per element, with matched amplitude taper.

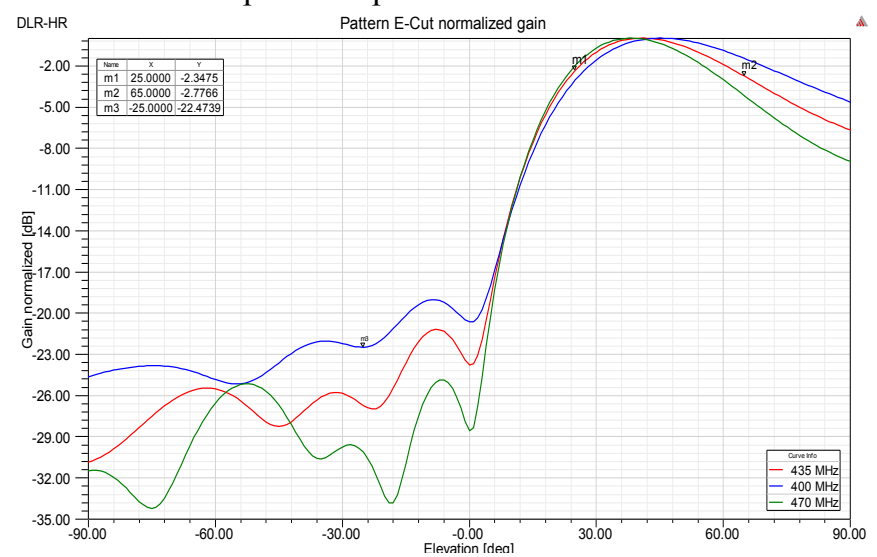

Fig. 6. Normalized one-way E-plane pattern in V-polarization at 400, 435 and $470 \mathrm{MHz}$

The reflections at each antenna port are reduced in relation to the former patch design, showing the good decoupling in the array of resonators.

The far field pattern indicates good steering capabilities and a smooth response to amplitude and phase tapering over the whole frequency range. Beam pointing is relatively constant in respect to the bandwidth. Especially the opposite swath suppression and nadir suppression is better than $22 \mathrm{~dB}$ for all frequencies, see Fig. 6.

In the desired radar application the pattern weights the reflected signals two times, thus the expected opposite swath suppression in raw data is about $40 \mathrm{~dB}$. It will be sufficient for 
most of the observed areas. Critical situations are low reflecting areas such as smooth water surfaces in the main region and man-made objects, like a village, in opposite swath direction. This configuration will still lead to ghosting effects in the image but cannot be neglected completely.

\section{OUTLOOK}

In the case of the P-band antenna the resonator box is part of mechanical structure of the antenna, guaranties at the same time electrical performance and structural stiffness. A prototype of reduces size will be manufactured. In a $3 \times 3$ elements array the behavior of the antenna will be tested. Not only electrical parameters will be measured but also structural tests will be performed in terms of airworthiness.

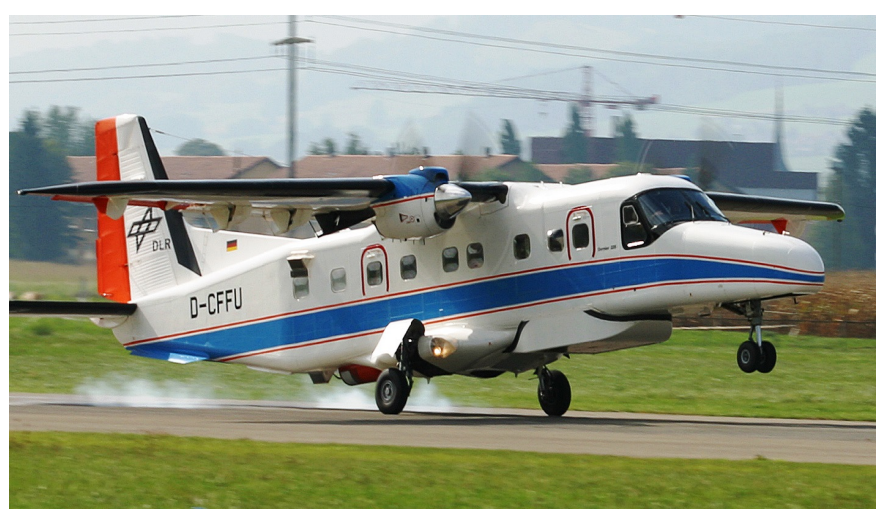

Fig. 7. One of the P-band antenna, mounted under the fuselage of a Dornier Do 228-212, in front of the main gear [4].

Critical topics in the manufacturing process will be investigated. It will be an indicator for manufacturing tolerances and material parameter variation. The important factor of overall weight of the antenna is combined with the single element design.

Due to the airworthiness process the mechanical design is very time consuming. Most of the material and components will be certified and tested for airborne use. The experiences from these tests will influence the design of the final flight model.

\section{CONCLUSION}

A new antenna type was tested to use in the F-SAR system. The low mutual coupling effects of the resonator backed patch element makes it extremely useful in electrically small arrays. It shows good performance and has advantages in airborne use where electrical and mechanical performance is crucial.

The goal is to extend the experience from the P-band antenna design to other frequency bands like L-band, where the resonator based element could by implemented with the methods of substrate integrated waveguides (SIW) techniques in an RF-multilayer board.

\section{REFERENCES}

[1] A. Kosc, "A 5 Way Lumped-Elements Wilkinson Power Divider", EuCAP 2013

[2] G. Mayhew-Ridgers, "Development and modelling of new wideband microstrip patch antennas with capacitive feed probes", University of Pretoria, 2004

[3] J. L. Vazquez-Roy, V. Krozer and Jorgen Dall, "Wideband dualpolarization mircrostrip patch antenna array for airborne ice sounder", IEEE Antennas and Propagation Magazine, vol. 54, no.4, Aug. 2012

[4] Dornier Do 228-212 D-CFFU of German Aerospace Center, at airliners.net, Photo from Renato Burkhart, 2003. 